Revista de Matemática: Teoría y Aplicaciones 2008 15(1) : 83-95

CIMPA - UCR ISSN: 1409-2433

\title{
CONFORMALLY INVARIANT DIFFERENTIAL OPERATORS AND BILINEAR FUNCTIONALS IN SIX DIMENSIONS
}

\author{
WilLiaM J. UGALDE*
}

Recibido/Received: 29 Jan 2007; Aceptado/Accepted: 31 Oct 2007

\begin{abstract}
We review how to construct the Paneitz operator in dimension four and the corresponding operator in dimension six, by constructing symmetric bilinear differential functionals that are conformally invariant.
\end{abstract}

Keywords: Conformal invariants, Paneitz operator, bilinear differential functionals.

\section{Resumen}

Revisamos como construir el operador de Paneitz en cuatro dimensiones y el correspondiente operador en seis dimensiones, mediante la construcción de funcionales diferenciales bilineales simétricos que son conformemente invariantes.

Palabras clave: Invariantes conformes, operador de Paneitz, funcionales bilineales diferenciales.

Mathematics Subject Classification: 53A30, 53-04.

\section{Introduction}

Using his quantized calculus, Connes [3] found a couple of conformal invariants on a fourdimensional oriented compact Riemannian manifold $M$ without boundary. The first one is a bilinear differential functional of order 4 , that we denote $B_{4}$ acting on $C^{\infty}(M)$. This bilinear differential functional is symmetric $B_{4}(f, h)=B_{4}(h, f)$, and conformally invariant in the sense that $\widehat{B_{4}}(f, h)=e^{-4 \eta} B_{4}(f, h)$, for a conformal change of the metric $\widehat{g}=e^{2 \eta} g$.

\footnotetext{
${ }^{*}$ Escuela de Matemática, Universidad de Costa Rica, 2060 San José, Costa Rica. Research partially supported by NSF grant DMS-9983601 and Vicerrectoría de Investigación de la Universidad de Costa Rica. E-Mail: wugalde@cariari.ucr.ac.cr
} 
The second conformal invariant, $P_{4}$, the Paneitz operator [8], can be derived derived from $B_{4}$ by the relation

$$
\int_{M} B_{4}(f, h) d x=\int_{M} f P_{4}(h) d x .
$$

These two conformal invariants can be defined in any even-dimensional oriented compact Riemannian manifold without boundary by the relations

$$
\operatorname{Wres}(k[F, f][F, h])=\int_{M} k B_{n}(f, h) d x, \quad \int_{M} B_{n}(f, h) d x=\int_{M} f P_{n}(h) d x,
$$

with $k, f, h \in C^{\infty}(M)$. For details in the four-dimensional case see [3] and for the general case see [12]. Here Wres is the non-commutative residue. The operator $F$ is a pseudodifferential operator of order 0 acting on the space of square integrable sections of middle dimension forms on $M$.

In this paper, we use Ricci.m [7], a package to do tensor computations to compute the conformal invariants $B_{4}$ and $P_{4}$ in the four-dimensional case, and the equivalent ones $B_{6}$ and $P_{6}$ in the six-dimensional conformally flat case. In detail we give a short introduction on how to use Ricci.m to do the computations required in this work. Next, we present our computation of $B_{4}$ and we prove Theorem 3.2 which characterizes any formally selfadjoint differential operator $P$ with leading term $\Delta^{2}$ and with no zeroth-order term which is furtermore conformal invariant as a constant multiple of the Paenitz operator. After that, we present our computation of $B_{6}$ in the conformally flat case and characterize in Theorem 3.5 all the symmetric bilinear differential functionals that are conformally invariant and that define a Hochschild 2-cocycle on the algebra $C^{\infty}(M)$ for a six-dimensional manifold $M$. At the end, we present Theorem 4.1 which characterizes all the differential operators in dimension six that are of type GJMS [5] as explained in Section 4.

In this work, $R$ represents Riemann tensor and the Ricci tensor is represented by $\mathrm{Rc}_{i j}=R^{k}{ }_{i k j}$. The scalar curvature by $\mathrm{Sc}=\mathrm{Rc}^{i}{ }_{i}$. We use $J$ to denote the normalized scalar curvature $2(n-1) J=\mathrm{Sc}$ in dimension $n$. If needed, we will "raise" and "lower" indices without explicit mention for example, $g_{m i} R_{j k l}^{i}=R_{m j k l}$. The relation between the Weyl tensor and the Riemann tensor is given by

$$
W_{j k l}^{i}=R^{i}{ }_{j k l}+\mathrm{P}_{j k} \delta_{K}{ }^{i}{ }_{l}-\mathrm{P}_{j l} \delta_{K}{ }^{i}{ }_{k}+\mathrm{P}^{i}{ }_{l} g_{j k}-\mathrm{P}^{i}{ }_{k} g_{j l}
$$

where $\delta_{K}$ represents the Kronecker's delta tensor. Last $(n-2) \mathrm{P}=\mathrm{Rc}-J g$.

\section{Working in a conformal setting with Ricci.m}

There are results supported on computations that are beyond reasonable hand manipulations. Introductions are presented in [1] with spin geometry and conformally invariant differential operators, and in [11] with gravitational field equations. Further examples are present in [6] with tractors, and in [9] and [10] with noise kernel for scalars fields in the Schwarzschild black hole and for thermal fields in flat space. This calculations require a package for tensor calculus with the capability of handling indices, covariant derivatives, 
curvature identities, symmetries, dependence on an arbitrary dimension, etc., and a particular kind of philosophy. The idea is to play an interactive session with a computer aiming to produce a desired result, not as the output of a program, but as the conclusion of a series of procedures, each based on a decision of what sort of identities or properties of the objects involved to use next. We use Ricci.m, a package prepared by J. M. Lee [7]. In what remains of this section we give a short presentation on how to use Ricci.m for the computations ahead.

We choose to work with the convention on the Laplacian having positive spectrum. To declare it in Ricci.m we must use

\section{\$LaplaceBeltramiConvention = PositiveSpectrum}

The first step is to define the bundle we will be working on:

\section{DefineBundle [tangent, n, , i, j, k,MetricType->Riemannian, RiemannConvention->FirstUp]}

where $\mathrm{g}$ represents the Riemannian metric associated to the base manifold of dimension $\mathrm{n}$ of the bundle tangent, and $i, j, k$ are indices associated to the bundle.

To be able to work with a conformal metric we must explicitly define a new metric gz which is conformal to the background metric $\mathrm{g}$. To define the new metric we use

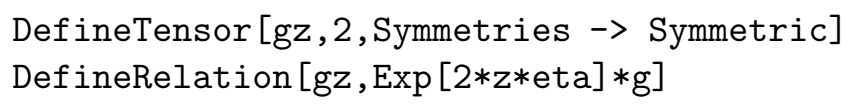

The $\mathbf{z}$ keeps track of the order of the conformal deformation or variation. For example, to compute the scalar curvature Sc in the new metric gz we use ScalarCurv[gz] which after simplifications with BasisExpand and TensorSimplify produces

$$
\operatorname{Sc}(g z)=\operatorname{Sc}(g) e^{-2 z \eta}-2(n-1) z \eta_{; i}{ }^{i} e^{-2 z \eta}-(n-2)(n-1) z^{2} \eta_{; i} \eta_{;}{ }^{i} e^{-2 z \eta} .
$$

It is very common to write $\widehat{g}$ for $g z=e^{2 z \eta} g, \widehat{S c}$ for $\operatorname{Sc}(g z)$, and simply Sc for $\operatorname{Sc}(g)$. In this way, the first order conformal variation of Sc is $-2(n-1) z \eta_{;} i^{i} e^{-2 z \eta}$ or simply $-2(n-1) \eta_{;}{ }^{i} e^{-2 \eta}$ if the metric is conformally changed to $e^{2 \eta} g$.

Before going any further is it important to make the following observation. If we set a metric $g$ in $M$ and consider another metric $\widehat{g}$ conformally related to $g$ by the relation $\widehat{g}=e^{2 z \eta} g$ where $\eta \in C^{\infty}(M)$ and $z$ a constant, then the conformal variation of each expression is a polynomial in $z$ whose coefficients are expressions in the metric and the conformal factor $\eta$ (actually, this is an abuse of the language since the conformal factor is $\left.e^{2 z \eta}\right)$. In this way, when studying conformal invariance, there is no need to study the whole conformal deformation. It is enough to study the conformal deformation up to order one in $\eta$ as follows. The conformal deformation up to order one in $\eta$ is given by $\left.\frac{d}{d z}\right|_{z=0}$. If a natural tensor or a differential operator is invariant up to order one in $\eta$, i.e. if its conformal deformation up to order one is equal to zero, then by integration it follows that it is fully invariant, for details see [2]. 
The next step to keep working with a conformal metric is to define its inverse Invgz. For that we use:

$$
\begin{aligned}
& \text { DefineTensor [Invgz, 2,Symmetries->Symmetric,Variance->Con] } \\
& \text { DefineRelation [Invgz [U[i], U[j] ],Exp[-2*z*eta]*g[U[i], U[j]]] }
\end{aligned}
$$

to declare it as a 2-contravariant symmetric tensor $g^{-1}$ such that $\left(g^{-1}\right)^{i j}=e^{-2 z \eta} g^{i j}$ and

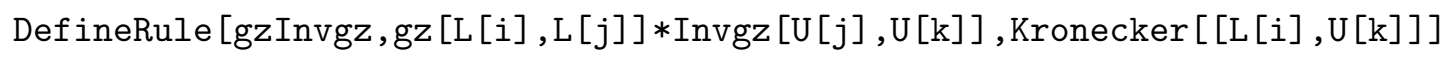

to define its properties as an inverse of $g$.

To declare smooth functions we consider them as 0 -tensors $f$ and $h$. Once they are declared using DefineTensor $[f, 0]$ and DefineTensor $[\mathrm{h}, 0]$ we can use the new metric to compute the conformal transformation of the different tensors we want to work with. For example, the conformal transformation of

$$
\begin{aligned}
f_{; i j} h_{;}^{i j} & =g^{i i_{1}} g^{j j_{1}} f_{; i j} h_{; i_{1} j_{1}} \\
& =\mathrm{g}[\mathrm{U}[\mathrm{i}], \mathrm{U}[\mathrm{i} 1]] * \mathrm{~g}[\mathrm{U}[\mathrm{j}], \mathrm{U}[\mathrm{j} 1]] * \mathrm{f}[][\mathrm{L}[\mathrm{i}], \mathrm{L}[\mathrm{j}]] * \mathrm{~h}[][\mathrm{L}[\mathrm{i} 1], \mathrm{L}[\mathrm{j} 1]]
\end{aligned}
$$

is obtained with

$\operatorname{Invgz}[\mathrm{U}[\mathrm{i}], \mathrm{U}[\mathrm{i} 1]] * \operatorname{Invgz}[\mathrm{U}[\mathrm{j}], \mathrm{U}[\mathrm{j} 1]] * \operatorname{Del}[\operatorname{Del}[f, \operatorname{Metric}->\mathrm{gz}]$, Metric->gz]

$[L[i], L[j]] * \operatorname{Del}[\operatorname{Del}[\mathrm{h}, \operatorname{Metric}>\mathrm{gz}], \operatorname{Metric->gz}][\mathrm{L}[\mathrm{i} 1], \mathrm{L}[\mathrm{j} 1]]$

\section{Computing $B_{4}$ and $B_{6}$.}

The bilinear differential form $B_{n}(f, h)$ is natural in the sense it can be written as a universal polynomial expression in the metric $g$, its inverse $g^{-1}$, the connection $\nabla$, and the curvature $R$; using tensor products and contractions. Furthermore, by Lemma 3.2 [12], we know that $B_{n}(f, h)$ is a polynomial in the ingredients $\nabla^{\alpha} d f, \nabla^{\beta} d h$, and $\nabla^{\gamma} R$ for multi-indices $\alpha, \beta$, and $\gamma$, with

$$
2 \operatorname{deg}_{R}+\operatorname{deg}_{\nabla}=n, \text { with } \operatorname{deg}_{\nabla} \geq 2 .
$$

Here for derivatives we count all of the covariant derivatives on $R, f$, and $h$, as well as the exterior derivatives, and any occurrence of $W, R c, \mathrm{P}, S c$, or $J$ is counted as an occurrence of $R$.

In the particular case $n=4, \operatorname{deg}_{R}$ can be 0 or 1 , hence $B_{4}$ can be written as

$$
B_{4}(f, h)=p_{4}(d f, d h)+p_{R, 4}(d f, d h)
$$

where $p_{R, 4}(d f, d h)$ is a trilinear form on $R, d f$, and $d h$. In the six-dimensional case, $\operatorname{deg}_{R} \in$ $\{0,1,2\}$ thus

$$
B_{6}(f, h)=p_{6}(d f, d h)+p_{6,1}(d f, d h)+p_{6,2}(d f, d h),
$$

with $p_{6,1}=p_{6, R}+p_{6, R^{\prime}}+p_{6, R^{\prime \prime}}$, and

1. $p_{6, R}(d f, d h)$ is polynomial on $\nabla^{\alpha} d f, \nabla^{\beta} d h$, and $R$, 
2. $p_{6, R^{\prime}}(d f, d h)$ is polynomial on $\nabla^{\alpha} d f, \nabla^{\beta} d h$, and $\nabla R$,

3. $p_{6, R^{\prime \prime}}(d f, d h)$ is polynomial on $\nabla^{\alpha} d f, \nabla^{\beta} d h$, and $\nabla \nabla R$, and

4. $p_{6,2}(d f, d h)$ is polynomial on $\nabla^{\alpha} d f, \nabla^{\beta} d h$, and $R R$.

The four-dimensional case was fully treated in [3], nevertheless we present our approach. By taking into consideration the symmetry of $B_{4}(f, h)$ we know

$$
p_{4}(d f, d h) \in \operatorname{span}\left\{\left(f_{; i}{ }^{i}{ }_{j} h_{;}{ }^{j}+h_{; i}{ }^{i}{ }_{j} f_{;}{ }^{j}\right), f_{; i j} h_{;}{ }^{i j}, f_{; i}{ }^{i} h_{; j}{ }^{j}\right\} \text {. }
$$

There are no terms in $p_{R, 4}(d f, d h)$ involving the Weyl tensor. Indeed, any term of the form $f_{; *} h_{; *} W_{* * * *}$ will force indices in $W$ to repeat and hence to vanish since the Weyl tensor is trace-free. In this way, the only terms to consider are those with a factor $\mathrm{P}$ or $J$. That is to say

$$
p_{R, 4}(f, h) \in \operatorname{span}\left\{f_{; i} h_{;}^{i} R_{j k}{ }^{j k}, f_{; i} h_{; j} R_{k}^{i}{ }^{j k}\right\}=\operatorname{span}\left\{f_{; i} h_{;}^{i} J, f_{; i} h_{; j} \mathrm{P}^{i j}\right\} .
$$

In particular, in the four-dimensional case, to determine $B_{4}(f, h)$ is enough to work in the conformally flat case. Thus, in the four dimensional case, $B_{4}(f, h)$ is a linear combination of the form:

$$
A\left(f_{; i}{ }_{i}{ }_{j} h_{;}^{j}+h_{; i}{ }^{i} f_{;}^{j}\right)+B f_{; i j} h_{;}^{i j}+C f_{; i}{ }^{i} h_{; j}{ }^{j}+D f_{; i} h_{;}^{i} J+E f_{; i} h_{; j} \mathrm{P}^{i j} .
$$

Its conformal variation, under a change of metric of the form $\widehat{g}=e^{2 z \eta} g$ with $\eta$ a smooth function on $M$, is given by:

$$
\begin{aligned}
& (2 C+B-2 A) z \eta_{; i}\left(f_{; j}{ }^{j} h_{;}{ }^{i}+f_{;}{ }^{i} h_{; j}{ }^{j}\right)+2(A-B) z \eta_{; i}\left(f_{; j}{ }^{i} h_{;}{ }^{j}+f_{; j} h_{;}{ }^{i j}\right) \\
& -D z \eta_{; i}{ }^{i} f_{; j} h_{;}^{j}+(4 A-E) z \eta_{; i j} f_{;}{ }^{i} h_{;}^{j} \\
& +(2 B-D-E / 2) z^{2} \eta_{; i} \eta_{;}^{i} f_{; j} h_{;}{ }^{j}+(2 B-8 A+4 C+E) z^{2} \eta_{; i} \eta_{; j} f_{;}{ }^{i} h_{;}{ }^{j} .
\end{aligned}
$$

Hence to get rid of the variation we just need to consider $B=A=2 C, E=4 A$, and $D=0$. Also note how the expressions with a $z^{2}$ factor vanish when the expressions with a $z$ factor vanish. In this way, by taking $C=-1$

$$
\begin{aligned}
B_{4}(f, h) & =-2\left(f_{; i}{ }^{i}{ }_{j} h_{;}{ }^{j}+h_{; i}{ }^{i}{ }_{j} f_{;}{ }^{j}\right)-2 f_{; i j} h_{;}{ }^{i j}-f_{; i}{ }^{i} h_{; j}{ }^{j}-8 f_{; i} h_{; j} \mathrm{P}^{i j} \\
& =-2\left(f_{; i j}{ }^{j} h_{;}{ }^{i}+h_{; i j}{ }^{j} f_{;}{ }^{i}\right)-2 f_{; i j} h_{;}{ }^{i j}-f_{; i}{ }^{i} h_{; j}{ }^{j}+4 f_{; i} h_{;}{ }^{i} J .
\end{aligned}
$$

By using the relations

$$
f_{; i}{ }^{i}=-\Delta f \quad f_{; i} h_{;}^{i}=\langle d f, d h\rangle \quad f_{; i j} h_{;}^{i j}=\langle\nabla(d f), \nabla(d h)\rangle
$$

and

$$
f_{; i j}{ }^{j} h_{;}^{i}+h_{; i j}{ }^{j} f_{;}^{i}=-\Delta\langle d f, d h\rangle-2\langle\nabla(d f), \nabla(d h)\rangle,
$$

in the four-dimensional case, $B_{4}$ is given by the following 
Theorem 3.1. For a four-dimensional oriented compact Riemanian manifold $M$ without boundary, any symmetric bilinear differential functional $B$ acting on $C^{\infty}(M) \times C^{\infty}(M)$, conformal invariant in the sense that $\widehat{B}(f, h)=e^{-4 \eta} B(f, h)$ for a conformal change of the metric $\widehat{g}=e^{2 \eta} g$, and such that $B(f, h)$ does not have linear terms on $f$ or $h$ is a constant multiple of

$$
B_{4}(f, h)=2 \Delta(\langle d f, d h\rangle)-\Delta(f) \Delta(h)+2\langle\nabla(d f), \nabla(d h)\rangle+4\langle d f, d h\rangle J .
$$

We use $\nabla$ to represent the covariant derivative and the Laplacian $\Delta=-\nabla_{j} \nabla^{j}$. Furthemore,

$$
\tau_{4}\left(f_{0}, f_{1}, f_{2}\right):=\int_{M} f_{0} B_{4}(f, h) d x
$$

defines a Hochschild 2-cocycle on the algebra of smooth functions on $M$.

The exclusion of linear terms on $f$ or $h$ in the statement is to avoid the presence of multiples of $f h|W|^{2}$. Compare with [3]. The cocycle property follows from an easy computation. What we have determined is actually $B_{4}$ up to a proportional factor. In [3], Connes use this expression for $B_{4}$ to obtain the Paneitz operator.

Theorem 3.2. Let $M$ be a four-dimensional oriented compact Riemanian manifold without boundary. Let $P$ be any formally selfadjoint differential operator $P$ with leading term $\Delta^{2}$ and with no zeroth-order term which is furtermore conformal invariant in the sense that $\widehat{P}(f)=e^{-4 \eta} P(f)$ for a conformal change of the metric $\widehat{g}=e^{2 \eta} g$. Then $P$ is a constant multiple of the Paenitz operator.

Proof. Stokes' theorem applied to (2) leads to an expression for $P_{4}$, indeed using the metric to raise and lower indices and reordering covariant derivatives

$$
\int_{M} B_{4}(f, h) d x=\int_{M}\left\{f_{; i}{ }^{i}{ }^{j}{ }^{j}-2 f_{; i}{ }^{i} J+2 f_{; i} J_{;}{ }^{i}+4 f_{; i j} \mathrm{P}^{i j}\right\} h d x .
$$

Because this equality holds for every $f$ and every $h$ in $C^{\infty}(M)$ we obtain,

$$
\begin{aligned}
P_{4}(f) & =f_{; i}{ }^{i}{ }^{j}{ }^{j}-2 f_{; i}{ }^{i} J+4 f_{; i j} \mathrm{P}^{i j}+2 f_{; i} J_{;}{ }^{i} \\
& =\Delta^{2}(f)+2 \Delta(f) J+4\langle\nabla d f \mathrm{P}\rangle+2\langle d f, d J\rangle,
\end{aligned}
$$

the Paneitz operator.

Now let $P$ be another formally selfadjoint differential operator of order 4 on the fourdimensional manifold $M$ such that $P$ is of the form $\Delta^{2}$ plus lower order terms and with no zeroth-order term. Define the symmetric bilinear differential functional $B$ by the relation

$$
B(f, h):=P(f h)-f P(h)-P(f) h .
$$

If $\widehat{P}(f)=e^{-4 \eta} P(f)$, for a conformal change of the metric $\widehat{g}=e^{2 \eta} g$ then, the same holds for $B$, that is $\widehat{B}(f, h)=e^{-4 \eta} B(f, h)$. Hence $B$ must be a constant multiple of $B_{4}$ above. Now

$$
\int_{M} f P_{4}(h) d x=\int_{M} B_{4}(f, h) d x=c \int_{M} B(f, h) d x=c \int_{M} f P(h) d x
$$


where the relation holds for any smooth functions $f$ and $h$ on $M$. Hence $P=c P_{4}$ and we have determined as well any possible differential operator of such a kind in the fourdimensional case up to a constant multiple.

In the 6-dimensional case, symmetry and conformal invariance are not enough to fully described $B_{6}$ as we will find terms like $f_{; i} h_{;}{ }^{i} W_{j k l m} W^{j k l m}$ and $f_{; i} h_{; j} W^{i}{ }_{k l m} W^{j k l m}$ which are symmetric on $f$ and $h$, and conformally invariant. We will need to use the Hochschild 2-cocycle property of $\operatorname{Wres}\left(f_{0}[F, f][F, h]\right)$ on the algebra $C^{\infty}(M)$ to determine some of the components of $B_{6}$. Even such a property will not suffice to completely describe $B_{6}$ as we will find terms that are conformally invariant and satisfy such a property (see (10)).

The list of terms we consider in the six-dimensional conformally flat case is as follows:

$$
\begin{aligned}
& p_{6}(d f, d h) \in \operatorname{span}\left\{\left(f_{; i} h_{; j}{ }^{j} k^{k i}+h_{; i} f_{; j}{ }^{j k i}{ }^{k i}\right),\left(f_{; i}{ }^{i} h_{; j}{ }^{j} k^{k}+h_{; i}{ }^{i} f_{; j}{ }^{j}{ }^{k}\right),\right. \\
& \left.\left(f_{; i j} h_{; k}{ }^{k i j}+h_{; i j} f_{; k}{ }^{k i j}\right),\left(f_{; i j k} h_{;}^{i j k}\right),\left(f_{; i}{ }^{i}{ }_{j} h_{; k}{ }^{k j}\right)\right\} \\
& p_{6, R}(d f, d h) \in \operatorname{span}\left\{\left(f_{; i} h_{; j}{ }^{j i}+h_{; i} f_{; j}{ }^{j i}\right) J,\left(f_{; i}{ }^{i} h_{; j}{ }^{j}\right) J,\left(f_{; i j} h_{;}{ }^{i j}\right) J,\right. \\
& \left(f_{; i} h_{; k}{ }^{k}{ }_{j}+h_{; i} f_{; k}{ }^{k}{ }_{j}\right) \mathrm{P}^{i j},\left(f_{; i} h_{; j k}^{i}+h_{; i} f_{; j k}^{i}\right) \mathrm{P}^{j k}, \\
& \left.\left(f_{; i j} h_{; k}^{k}+h_{; i j} f_{; k}^{k}\right) \mathrm{P}^{i j},\left(f_{; i j} h_{; k}^{i}\right) \mathrm{P}^{j k}\right\}
\end{aligned}
$$

Next, for $p_{6, R^{\prime}}$ we might consider

$$
\begin{aligned}
p_{6, R^{\prime}} \in \operatorname{span}\{ & \left(f_{; i} h_{; j}{ }^{j}+h_{; i} f_{; j}{ }^{j}\right) J_{;}{ }^{i},\left(f_{; i} h_{; j}{ }^{i}+h_{; i} f_{; j}{ }^{i}\right) J_{;}{ }^{j}, \\
& \left(f_{; i j} h_{; k}+h_{; i j} f_{; k}\right) \mathrm{P}^{i j}{ }^{k},\left(f_{; i j} h_{; k}+h_{; i j} f_{; k}\right) \mathrm{P}^{i k}{ }^{j}, \\
& \left.\left(f_{; i j} h_{;}{ }^{i}+h_{; i j} f_{;}{ }^{i}\right) \mathrm{P}^{j}{ }_{k ;}{ }^{k},\left(f_{; i j} h_{;}{ }^{i}+h_{; i j} f_{;}{ }^{i}\right) \mathrm{P}_{k}{ }^{k}{ }_{;}^{j}\right\} .
\end{aligned}
$$

The relations

$$
\mathrm{P}_{i}^{i}=J, \quad \mathrm{P}_{i j}{ }_{;}^{j}=J_{; i}, \quad \mathrm{P}_{i j ; k}=\mathrm{P}_{i k ; j}-W_{i l j k}{ }_{;}^{l} /(n-3)
$$

imply that these terms are already considered in the conformally flat case and hence

$$
p_{6, R^{\prime}} \in \operatorname{span}\left\{\left(f_{; i} h_{; j}{ }^{j}+h_{; i} f_{; j}{ }^{j}\right) J_{;}^{i},\left(f_{; i} h_{; j}^{i}+h_{; i} f_{; j}^{i}\right) J_{;}{ }^{j},\left(f_{; i j} h_{; k}+h_{; i j} f_{; k}\right) \mathrm{P}^{i j}{ }_{;}{ }^{k}\right\} .
$$

For $p_{6, R^{\prime \prime}}$ we might consider

$$
\begin{aligned}
\operatorname{span}\{ & f_{; i} h_{;}{ }^{i} J_{; j}{ }^{j}, f_{; i} h_{; j} J_{;}^{i j}, f_{; i} h_{; j} \mathrm{P}^{i j}{ }_{; k}{ }^{k}, f_{; i} h{ }_{;}{ }^{i} \mathrm{P}_{j k}{ }_{; k}{ }^{j k}, \\
& \left.\left(f_{; i} h_{; j}+h_{; i} f_{; j}\right) \mathrm{P}_{i k}{ }^{j k},\left(f_{; i} h_{; j}+h_{; i} f_{; j}\right) \mathrm{P}^{i j}{ }_{; k}{ }^{k}\right\} .
\end{aligned}
$$

The relation

$$
\begin{aligned}
\mathrm{P}_{i j ; l k}-\mathrm{P}_{i j ; k l} & =\mathrm{P}_{j}{ }^{m}\left(g_{l m} \mathrm{P}_{i k}-g_{k m} \mathrm{P}_{i l}-g_{i l} \mathrm{P}_{k m}+g_{i k} \mathrm{P}_{l m}+W_{i m k l}\right) \\
& +\mathrm{P}_{i}{ }^{m}\left(g_{l m} \mathrm{P}_{j k}-g_{k m} \mathrm{P}_{j l}-g_{j l} \mathrm{P}_{k m}+g_{j k} \mathrm{P}_{l m}+W_{j m k l}\right)
\end{aligned}
$$

and (5) above imply

$$
p_{6, R^{\prime \prime}} \in \operatorname{span}\left\{f_{; i} h_{;}^{i} J_{; j}{ }^{j}, f_{; i} h_{; j} J_{;}^{i j}\right\}
$$


Last,

$$
p_{6, R^{2}} \in \operatorname{span}\left\{f_{; i} h_{;}^{i} J^{2}, f_{; i} h_{; j} J \mathrm{P}^{i j}, f_{; i} h_{;}{ }^{i} \mathrm{P}_{j k} \mathrm{P}^{j k}, f_{; i} h_{; j} \mathrm{P}_{k}^{i} \mathrm{P}^{j k}\right\} .
$$

In this way, we know $B_{6}(f, h)$ in the conformally flat case is a linear combination of up to 21 terms. We use Ricci.m to compute the proper coefficients, the result reads:

Theorem 3.3. In the 6-dimensional conformally flat case, an expression for $B_{6}$ is

$$
\begin{aligned}
& B_{6, \text { confflat }}(f, h)=2\left(f_{; i}{ }^{i}{ }_{j}{ }_{k} h_{;}{ }^{k}+h_{; i}{ }^{i}{ }_{j}{ }_{k} f_{;}{ }^{k}\right)+\left(f_{; i}{ }^{i}{ }_{j}{ }^{j} h_{; k}{ }^{k}+h_{; i}{ }^{i}{ }^{j} f_{; k}{ }^{k}\right) \\
& +4\left(f_{; i}{ }^{i}{ }_{j k} h_{;}{ }^{j k}+h_{; i}{ }^{i}{ }_{j k} f_{;}{ }^{j k}\right)+4 f_{; i}{ }^{i} h_{; k}{ }^{k j}+8\left(f_{; i j k} h_{;}{ }^{i j k}\right) / 3 \\
& -4\left(f_{; i}{ }^{i}{ }_{j} h_{;}{ }^{j}+h_{; i}{ }^{i}{ }_{j} f_{;}{ }^{j}\right) J-4 f_{; i}{ }^{i} h_{; j}{ }^{j} J \\
& +80\left(f_{; i} h_{; j}{ }^{j} k+h_{; i} f_{; j}{ }^{j}\right) \mathrm{P}^{i k} / 3 \\
& +32\left(f_{; i j k} h_{;}^{i}+f_{; i j k} h_{;}^{i}\right) \mathrm{P}^{j k} / 3+64 f_{; i j} h_{;}{ }^{i k} \mathrm{P}^{j k} \\
& +8\left(f_{; i j} h_{;}{ }^{i}+h_{; i j} f_{;}^{i}\right) J_{;}{ }^{j}+32\left(f_{; i j} h_{; k}+h_{; i j} f_{; k}\right) \mathrm{P}^{i j}{ }_{;}^{k} \\
& +16 f_{; i} h_{; j} J_{;}^{i j} \\
& -32 f_{; i} h_{; j} J \mathrm{P}^{i j}-80 f_{; i} h_{;}{ }^{i} \mathrm{P}_{j k} \mathrm{P}^{j k} / 3+\left(608 f_{; i} h_{; j} \mathrm{P}_{k}^{i} \mathrm{P}^{j k}\right) / 3 .
\end{aligned}
$$

It is not difficult to verify that the above expression for $B_{6}$ in the conformally flat case, as a sum of explicitely symmetric terms is:

$$
\begin{aligned}
& B_{6, \text { confflat }}(f, h) \\
& =2\left(\left\langle d \Delta^{2} f, d h\right\rangle+\left\langle d \Delta^{2} h, d f\right\rangle\right)-\left(\Delta^{2} f \Delta h+\Delta^{2} h \Delta f\right) \\
& -4(\langle\nabla d \Delta f, \nabla d h\rangle+\langle\nabla d \Delta h, \nabla d f\rangle)+4\langle d \Delta f, d \Delta h\rangle+8\left\langle\nabla^{2} d f, \nabla^{2} d h\right\rangle / 3 \\
& +4(\langle d \Delta f, d h\rangle+\langle d f, d \Delta h\rangle) J-4 \Delta f \Delta h J \\
& -80\langle d f \otimes d \Delta h+d h \otimes d \Delta f, \mathrm{P}\rangle / 3+32\left\langle\nabla^{2} d f, d h \otimes \mathrm{P}\right\rangle \\
& +\left\langle\nabla^{2} d h, d f \otimes \mathrm{P}\right\rangle / 3+64\langle\nabla d\langle d f, d h\rangle, \mathrm{P}\rangle \\
& -32\left(\left\langle\nabla^{2} d f, d h \otimes \mathrm{P}\right\rangle+\left\langle\nabla^{2} d h, d f \otimes \mathrm{P}\right\rangle\right) \\
& +8(\langle\nabla d f, d h \otimes d J\rangle+\langle\nabla d h, d f \otimes d J\rangle) \\
& +32(\langle\nabla d f \otimes d h+\nabla d h \otimes d f, \nabla V\rangle)+16\langle d f \otimes d h, \nabla d J\rangle \\
& -32\langle d f \otimes d h, \mathrm{P}\rangle J-80\langle d f, d h\rangle\langle\mathrm{P}, \mathrm{P}\rangle / 3+\left(608\left\langle d f \otimes d h, V^{2}\right\rangle\right) / 3 .
\end{aligned}
$$

Here we are using $\left\langle d f \otimes d h, V^{2}\right\rangle$ to denote $f_{; i} h_{; j} \mathrm{P}_{k}{ }_{k} \mathrm{P}^{j k}$.

Leaving for an instant the conformally flat case, in the general conformally curved case, the conformal variation of $B_{6}(f, h)$ in $(6)$, up to order one in $\eta$ is given by

$$
-64 z \eta_{; i}\left(f_{; j k} h_{; l}+f_{; l} h_{; k j}\right) W^{i j k l} / 3-32 z \eta_{; i j} f_{; k} h_{; l} W^{i l j k}+160 z^{2} \eta_{; i} \eta_{; j} f_{; k} h_{; l} W^{i k j l} / 3 .
$$

which vanishes in the conformally flat case, meaning that our expression is conformally invariant inside the conformally flat class of metrics on $M$. In the general conformally curved case, this variation will be useful in finding the extra terms we are missing, that is to say, those terms that vanish in the conformally flat case.

If we define using (6) the trilinear form on $C^{\infty}(M)$

$$
\tau\left(f_{0}, f_{1}, f_{2}\right):=\int_{M} f_{0} B_{6}\left(f_{1}, f_{2}\right) d x
$$


then

$$
\begin{aligned}
& (b \tau)\left(f_{0}, f_{1}, f_{2}, f_{3}\right) \\
& =\int_{M} f_{0}\left(32 f_{1 ; i} f_{2 ; j} f_{3 ; k} W^{i k} l_{;}^{j}+32\left(f_{1 ; i j} f_{3 ; l}-f_{1 ; l} f_{3 ; i j}\right) f_{2 ; k} W^{i k j l} / 3\right) d x
\end{aligned}
$$

which vanishes in the conformally flat case meaning that $\tau$ is a Hochschild 2-cocycle on the algebra of smooth functions in the conformally flat case.

We do not restrict ourselves anymore to the conformally flat case. Our objective now is to identify those terms that we must add to $p_{6, R}, p_{6, R^{\prime}}, p_{6, R^{\prime \prime}}$, and $p_{6,2}$ (in (6)) to obtain the expression for $B_{6}$ in the general conformally curved case. In particular, we are looking for terms having the Weyl tensor, or its covariant derivatives as factors.

The first set of terms to be added will complete the expression for $p_{6, R}$. In this case there is just one possibility which is $f_{; i j} h_{; k l} W^{i k j l}$. Any other possibility is ruled out by the Bianchi identities. To complete the expression for $p_{6, R^{\prime}}$ we only need to consider $\left(f_{; i j} h_{; k}+\right.$ $\left.h_{; i j} f_{; k}\right) W^{i} l^{j k}{ }_{;}$. The only term to complete the expression for $p_{6, R^{\prime \prime}}$ is $f_{; i} h_{; j} W^{i} k_{k}{ }_{l}{ }^{k l}$, symmetric on $f$ and $h$. For $p_{6,2}$ we consider at this time only $f_{; i} h h_{; j} \mathrm{P}_{k l} W^{i k j l}$. It happens that the other possible terms

$$
f_{; i} h_{;}{ }^{i} W_{j k l m} W^{j k l m} \quad \text { and } \quad f_{; i} h_{; j} W_{k l m}^{i} W^{j k l m} .
$$

are conformally invariant.

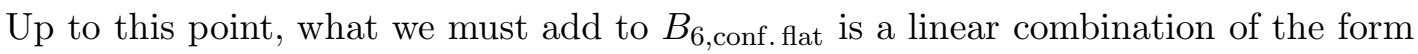

$$
A f_{; i j} h_{; k l} W^{i k j l}+B\left(f_{; i j} h_{; k}+h_{; i j} f_{; k}\right) W^{i} l_{; k}^{l}+C f_{; i} h_{; j} W_{k}^{i}{ }^{j}{ }_{;}^{k l}+D f_{; i} h_{; j} \mathrm{P}_{k l} W^{i k j l} .
$$

Its conformal variation up to order one in $\eta$ is given by

$$
\begin{aligned}
& (2 A-3 B) z \eta_{; i}\left(f_{; j k} h_{; l}+h_{; j k} f_{; l}\right) W^{i j k l}+(3 C-D) z \eta_{; i j} f_{; k} h_{; l} W^{i l j k} \\
& -(B+2 C) z \eta_{; i} f_{; j} h_{; k}\left(W^{i j k}{ }_{l}+W^{i k j}{ }_{l}\right)_{;}^{l}-(2 A-6 B-3 C-D) z^{2} \eta_{; i} \eta_{; j} f_{; k} h_{; l} W^{i k j l} .
\end{aligned}
$$

By comparing it with (8), we deduce the conditions

$$
B+2 C=0, \quad 2 A-3 B=64 / 3, \quad 3 C-D=32,
$$

which means, conformally invariance and symmetry are not enough to find the right values for all the constants. So far, the term to be added to $B_{6}(f, h)$ is given by

$$
\begin{aligned}
& (32 / 3-3 C) f_{; i j} h_{; k l} W^{i k j l}-2 C\left(f_{; i j} h_{; k}+h_{; i j} f_{; k}\right) W_{l}^{i} l_{; k}{ }_{;}+C f_{; i} h_{; j} W_{k}^{i}{ }_{k}{ }_{l}{ }^{k l} \\
& +3 C f_{; i} h_{; j} \mathrm{P}_{k l} W^{i k j l}+E f_{; i} h_{;}{ }^{i} W_{j k l m} W^{j k l m}+F f_{; i} h_{; j} W^{i}{ }_{k l m} W^{j k l m}
\end{aligned}
$$

where the last two terms come from (10).

In the 4-dimensional case, the Hochschild 2-cocycle property of $\operatorname{Wres}\left(f_{0}\left[F, f_{1}\right]\left[F, f_{2}\right]\right)$ was used merely to make sure the constants found had the right values. In the 6dimensional case, as we shall see, this property will play a more important role in the non-conformally flat case. Nevertheless, the full description of $B_{6}$ escapes even these properties, requiring some more information to be used. 
Proposition 3.4. The trilinear form

$$
\begin{aligned}
& \tau^{\prime}\left(f_{0}, f_{1}, f_{2}\right):= \\
& \int_{M} f_{0}\left\{f_{1 ; i} f_{2 ; j}\left(C W_{k}^{i}{ }_{k}{ }^{k}{ }^{k l}+D \mathrm{P}_{k l} W^{i k j l}+F W_{k l m}^{i} W^{j k l m}\right)+E f_{1 ; i} f_{2}{ }^{i} W_{j k l m} W^{j k l m}\right\} d x
\end{aligned}
$$

defines a Hochschild 2-cocycle on the algebra $C^{\infty}(M)$ for any value of the constants $C, D$, $E$, and $F$, that is, it satisfies $\left(b \tau^{\prime}\right)\left(f_{0}, f_{1}, f_{2}, f_{3}\right)=0$ for any $f_{i} \in C^{\infty}(M)$.

On the other hand, if we define

$$
\tau^{\prime \prime}\left(f_{0}, f_{1}, f_{2}\right):=\int_{M} f_{0}\left\{A f_{1 ; i j} f_{2 ; k l} W^{i k j l}+B\left(f_{1 ; i j} f_{2 ; k}+f_{2 ; i j} f_{1 ; k}\right) W_{l}^{i} l_{; k}^{l}\right\} d x
$$

then

$$
\begin{aligned}
& \left(b \tau^{\prime \prime}\right)\left(f_{0}, f_{1}, f_{2}, f_{3}\right)= \\
& \int_{M} f_{0}\left(3 B f_{1 ; i} f_{2 ; j} f_{3 ; k} W^{i k} l^{j}{ }^{l}+2 A\left(f_{1 ; i j} f_{3 ; l}-f_{1 ; l} f_{3 ; i j}\right) f_{2 ; k} W^{i k j l}\right) d x .
\end{aligned}
$$

To have that $\int_{M} f_{0} B_{6}\left(f_{1}, f_{2}\right) d x$ is a Hochschild 2-cocycle on $C^{\infty}(M)$ we need $(9)+(13)=$ 0 , for any $f_{i} \in C^{\infty}(M)$. Thus $3 B=-32$ and $3 A=-16$, which agree with (11). We must also have $3 C=16$ and $D=-16$. Hence using (6), (10), and (12) we conclude the following expression for $B_{6}(f, h)$ :

$$
\begin{aligned}
B_{6}(f, h) & =B_{6, \text { confflat }}(f, h)-16 f_{; i j} h_{; k l} W^{i k j l} / 3-32\left(f_{; i j} h_{; k}+h_{; i j} f_{; k}\right) W_{l}^{i j k}{ }_{;}^{l} / 3 \\
& +16 f_{; i} h_{; j} W_{k}^{i}{ }_{k} l_{;}{ }^{k l} / 3+16 f_{; i} h_{; j} \mathrm{P}_{k l} W^{i k j l} \\
& +E f_{; i} h_{;}^{i} W_{j k l m} W^{j k l m}+F f_{; i} h_{; j} W^{i}{ }_{k l m} W^{j k l m}
\end{aligned}
$$

where the last two terms are the needed ones to fully complete the expression for $p_{6,2}$ as in (10). Here it is important to keep in mind that one may have many different expressions for the same object, furthermore different expressions that yield the same invariant on conformally flat structures, may represent different invariants in more curved settings. In this particular case, any value of $E$ or $F$ will yield the same invariant in the conformally flat case.

Theorem 3.5. The expression (14) gives a family of bilinear differential functionals acting on $C^{\infty}(M) \times C^{\infty}(M)$ (without order zero terms) of order 6 associated to a six-dimensional compact oriented Riemannian manifold $M$ without boundary. Each of these differential functionals is symmetric on $f$ and $h$, conformally invariant in the sense that $\widehat{B_{6}}(f, h)=$ $e^{-6 \eta} B_{6}(f, h)$, for a conformal change of the metric $\widehat{g}=e^{2 \eta} g$, and defines a Hochschild 2-cocycle on the algebra $C^{\infty}(M)$ by the relation $\tau\left(f_{0}, f_{1}, f_{2}\right)=\int_{M} f_{0} B_{6}\left(f_{1}, f_{2}\right) d x$.

Finding the right values of $E$ and $F$ to obtain in the 6-dimensional conformally curved case the unique bilinear differential functional satisfying the relation

$$
\operatorname{Wres}\left(f_{0}\left[F, f_{1}\right]\left[F, f_{2}\right]\right)=\int_{M} f_{0} B_{6}\left(f_{1}, f_{2}\right) d x
$$

will be the topic of a different paper. 


\section{The analogue of the Paneitz operator in six dimensions}

Stokes' theorem applied to (14) leads to the expression

$$
\operatorname{Wres}([F, f][F, h])=\int_{M} P_{6}(f) h d x
$$

(see [12] for details). Because this equality holds for every $f$ and every $h$ in $C^{\infty}(M)$ we obtain an operator $P_{6}$ constructed in a canonical way from the Wodzicki residue Wres $([F, f][F, h])$. In the flat case we obtain simply

$$
P_{6 \text { flat }}(f)=2 \Delta^{3}(f) / 3=-2 f_{; i} i{ }_{j}{ }_{k} k / 3 \text {. }
$$

In the conformally flat case we have terms with $\nabla^{a} J \nabla^{b} V$ as a factor:

$$
\begin{aligned}
& P_{6 \text { conf. flat }}(f)=P_{6 \text { flat }}(f) \\
& +4 h_{; i}{ }_{i j}{ }^{j} J-32 / 3 h_{; i}{ }_{j k} \mathrm{P}^{j k}-8 / 3 h_{; i}{ }_{i}{ }_{j} J_{;}{ }^{j}-32 / 3 h_{; i j k} \mathrm{P}^{i j} ; \\
& +h_{; i}{ }^{i}\left(4 / 3 J_{; j}{ }^{j}-16 / 3 J^{2}-32 / 3 \mathrm{P}_{j k} \mathrm{P}^{j k}\right) \\
& +h_{; i j}\left(64 / 3 J_{;}{ }^{i j}+64 / 3 J \mathrm{P}^{i j}+64 \mathrm{P}^{i}{ }_{k} \mathrm{P}^{j k}-32 \mathrm{P}^{i j}{ }_{; k}{ }^{k}\right) \\
& +h_{; i}\left(32 / 3 J J_{;}{ }^{i}-8 / 3 J_{; j}{ }^{j i}-128 / 3 J_{; j} \mathrm{P}^{i j}-128 / 3 \mathrm{P}_{j}{ }^{k} \mathrm{P}^{i j}{ }_{;}{ }^{k}\right) .
\end{aligned}
$$

In the general conformally curved case, we also those terms with a Weyl tensor as a factor:

$$
\begin{aligned}
& P_{6}(f)=P_{6 \text { conf. flat }}(f) \\
& -16 / 3 h_{; i j k l} W^{i l j k}-16 h_{; i j k} W^{i k j}{ }_{l ;}{ }^{l}-E h_{; i}{ }^{i} W_{j k l m} W^{j k l m} \\
& +h_{; i j}\left(64 / 9 W^{i}{ }_{k}{ }_{l}{ }^{k l}-(8 / 3+F) W^{i}{ }_{k l m} W^{j k l m}-80 / 3 \mathrm{P}_{k l} W^{i k j l}\right) \\
& +h_{; i}\left(80 / 9 \mathrm{P}_{j k} W^{i j k}{ }_{l}{ }_{;}{ }^{i}-104 / 3 \mathrm{P}_{j k}{ }_{j l} W^{i j k}-2 E W_{j k l m} W^{j k l m}{ }^{i}\right. \\
& \left.\quad-2(44+3 F) / 3 W^{i}{ }_{j k l} W_{m}^{j k l}{ }_{m}^{m}+52 / 3 W_{j k l m} W_{;}^{i j k l}{ }^{m}+(34+3 F) W_{j k l m} W^{i j l m}{ }_{;}^{k}\right) .
\end{aligned}
$$

The GJMS operators [5] are invariant operators on conformal densities

$$
P_{2 k}: \mathcal{E}[-n / 2+k] \rightarrow \mathcal{E}[-n / 2-k]
$$

with principal parts $\Delta^{k}$, unless the dimension is even and $2 k>n$. If $n$ is even, the $n$-th order operator

$$
P_{n}: \mathcal{E}[0] \rightarrow \mathcal{E}[-n]
$$

is called critical GJMS operator. As noted in [5], $\mathcal{E}[0]=C^{\infty}(M)$ and $\mathcal{E}[-n]$ is the bundle of volume densities on $M$. In the 4-dimensional case, the Paneitz operator coincide with the critical GJMS operator. The GJMS operators $P_{2 k}$, of Graham-Jenne-Mason-Sparling [5] by construction have the following properties.

i. $P_{2 k}$ exists for all $k$ if $n$ is odd, and if $n$ is even exists for $1 \leq k \leq n / 2$.

ii. $P_{2 k}$ is formally self-adjoint. 
iii. $P_{2 k}$ is conformally invariant in the sense that

$$
\widehat{P}_{2 k}=e^{-(n / 2+k) \eta} P_{2 k} e^{(n / 2-k) \eta}
$$

for conformally related metrics $\widehat{g}=e^{2 \eta} g$.

iv. $P_{2 k}$ has a polynomial expression in $\nabla$ and $R$ in which all coefficients are rational in the dimension $n$.

v. $P_{2 k}=\Delta^{k}+$ lot. (Here and below, lot $=$ "lower order terms".)

vi. $P_{2 k}$ has the form

$$
\delta S_{2 k} d+\left(\frac{n}{2}-k\right) Q_{2 k}
$$

where $Q_{2 k}$ is a local scalar invariant, and $S_{2 k}$ is an operator on 1-forms of the form

$$
(d \delta)^{k-1}+\text { lot or } \Delta^{k-1}+\text { lot } .
$$

In this last expression, $d$ and $\delta$ are the usual de Rham operators and $\Delta$ is the form Laplacian $d \delta+\delta d$. The original work [5] uses the "ambient metric construction" of [4] to prove their existence.

Theorem 4.1. Any polynomial $P_{6}$ of the form (15) is conformally invariant and a critical GJMS operator (in the sense that it satisfies the properties $i$. to vi. above) in a six dimensional, compact, conformal manifold without boundary.

A computation verifies the following particular six dimensional case of Theorem 1.2.vi. of $[12]$

Proposition 4.2. The functional $B_{6}$ and the polynomial $P_{6}$ are related by

$$
P_{6}(f h)=P_{6}(f) h+f P_{6}(h)-2 B_{6}(f, h) .
$$

This relation opens the gate to study the relation between products of GJMS-operators of order different than the critical and bilinear differential functionals. Relation that we will explore in future work.

\section{References}

[1] Branson, T.P. (2001) "Automated symbolic computations in spin geometry", in: F. Brackx, J.S.R. Chisholm \& V. Souček (Eds.) Clifford Analysis and its Applications, NATO Science Series II, Vol. 25, Kluwer Academic Publishers, Dordrecht: 27-38.

[2] Branson, T.P. (1985) "Differential operators canonically associated to a conformal structure", Math. Scand. 57: 293-345.

[3] Connes, A. (1995) "Quantized calculus and applications", in: Proceedings of the XIth International Congress of Mathematical Physics, International Press, Cambridge, MA: 15-36. 
[4] Fefferman, C.; Graham, C.R. (1985) Conformal Invariants. In Élie Cartan et les mathématiques d'aujourd'hui, Astérisque, hors série, Société Mathé- matique de France: $95-116$.

[5] Graham, R.; Jenne, R.; Mason, L.; Sparling, G. (1992) "Conformally invariant powers of the Laplacian, I: Existence", J. London Math. Soc. (2) 46: 557-565.

[6] Gover, A. R.; Peterson, L.J. (2003) "Conformally invariant powers of the Laplacian, Q-curvature and tractor calculus", Comm. Math. Phys. 235(2): 339-378.

[7] Lee, J.M. (s.f.) "A Mathematica package for doing tensor calculations in differential geometry", available at http://www.math.washington.edu/ lee/Ricci/.

[8] Paneitz, S. (1983) "A quartic conformally covariant differential operator for arbitrary pseudo-Riemannian manifolds", preprint, Massachusetts Institute of Technology.

[9] Phillips, N.G.; Hu, B.L. (2003) "Noise kernel and stress energy bi-tensor of quantum fields in conformally-optical metrics: Schwarzschild black holes", Phys. Rev. D (3) 67(10) 104002, 26 pp.

[10] Phillips, N.G.; Hu, B.L. (2001) "Noise kernel and stress energy bi-tensor of quantum fields in hot flat space and Gaussian approximation in the optical Schwarzschild metric", Phys. Rev. D (3) 63(10) 104001, 16 pp.

[11] Tsantalis, E.; Puntigam, R.A.; Hehl, F.W. (1996) "A quadratic curvature Lagrangian of Pawlowski and Raczka: a finger exercise with math tensor", Relativity and Scientific Computing, Springer, Berlin: 231-240.

[12] Ugalde, W.J. (2006) "A construction of critical GJMS operators using Wodzicki's residue" Comm. Math. Phys. 261(3): 771-788. 